\title{
news news news news news news news news
}

\section{Africa initiative: Online resources on mental health and HIV/AIDS}

As part of its Mental Health Africa initiative the World Federation for Mental Health (WFMH) has launched three new online resource directories to provide access to reference materials, reports, and best practices on mental health and psychosocial support programmes addressing the mental health consequences of HIV/AIDS. These cover resources on mental health and HIV/AIDS, psychosocial support /reports, resources and best practices), and psychosocial support organisations. The development of these online directories was recommended by the participants in the WFMH's International Experts Forum addressing the mental health consequences of HIV/AIDS held in Cape Town, South Africa in January.

The WFMH has also launched a global online directory of mental health patient/service user, family member/caregiver support groups, and mental health advocacy and awareness organisations

Source: www.wfmh.org

\section{Intellectual disabilities surveyed}

While almost $60 \%$ of African countries have legislation to protect persons with intellectual disabilities, only $40 \%$ have a judicial protection system for children and adults and $37 \%$ a protection system for adults. These are among the findings of the first Atlas of Global Resources for Persons with Intellectual Disabilities, which was compiled by the World Health Organization (WHO) and the Montreal PAHO/WHO Collaborating Centre for Research and Training in Mental Health. Its aim is to improve evidence-based knowledge and awareness on global and regional disparities through information on the resources and services for persons with intellectual disability at country level.

The most common diagnostic systems used are the International Classification of Diseases (ICD-10) and the Diagnostic and Statistical Manual of Mental Disorders (DSM-IV), although in Africa about half of countries also rely on professional opinion and clinical judgement.

Within Africa the main source of funding for services is out-ofpocket expenses and NGOs, compared with the taxed-based funding in much of the rest of the world. Moreover, just over half countries in Africa do not provide state benefits and in the majority of those that do the benefits are low.

Globally the Atlas finds that intellectual disabilities have low visibility and low priority on a number of national political, social and economic agendas, and in some circumstances coverage in generic policies, laws, or programmes can remain largely theoretical. While most countries appear to offer some sort of service for intellectual disabilities, access remains critical with fewer than $10 \%$ of those eligible receiving government benefits in almost $40 \%$ of countries.

Recommendations include making intellectual disabilities a public health issue and recognising the crucial support role of families, in addition to changing the priorities of governments and civil society and scaling up funding for these disorders.

Source: www.who.int

\section{New diploma in mental health law and human rights}

The Indian Law Society in collaboration with the WHO's Mental Health Improvement for Nations Development (MIND) project has developed the first 1-year international diploma in mental health law and human rights. The course, based on WHO MIND materials and tools, provides students with the opportunity to develop essential knowledge and expertise in the area of mental health, human rights and law. By the end of the course students will have acquired the skills necessary for supporting countries to put in place human rights-oriented mental health laws and policies, and the confidence to effectively advocate for improved conditions for people with mental disabilities.

The course comprises both residential sessions and distance learning, and will be taught and supervised by a faculty comprising renowned experts in the area of mental health, human rights and legislation from around the globe.

The course starts on 13 October 2008, beginning with a series of onsite lectures at the ILS Law College, Pune, India. Applications to participate are now being considered.

Source: www. mentalhealthlaw.in/index.html

\section{World Mental Health Day: Scaling up services through advocacy and action}

World Mental Health Day 2008 will take place on 10 October under the theme 'Making mental health a global priority: Scaling up services through citizen advocacy and action'.

With the final goal of making mental health a global priority in every country, what needs to be changed or what action needs to be taken in order to fulfil that goal?. Highlighted will be different levels of advocacy and its role in creating change and the need for scaling up services so all people have access to information, personalised treatment, and resources to assist them in all aspects of their recovery.

Source: www.wfmh.org 